\title{
A STUDY OF RISK FACTORS IN YOUNG PATIENTS OF ACUTE CORONARY SYNDROME
}

\author{
BISWAS PK ${ }^{1}$, SARDAR MH ${ }^{2}$,SAHA GC ${ }^{3}$, HOSSAIN MZ ${ }^{4}$, HOSSAIN MS ${ }^{5}$, PAUL T ${ }^{6}$, AZAD KAK ${ }^{7}$
}

\begin{abstract}
:
Aim: The study was carried out to see the risk factors of Acute Coronary Syndrome in young patients.

Materials and Methods: This is a prospective study which was carried out in 60 young patients (18-40 years of age) with a diagnosis of Acute Coronary Syndrome admitted to Dhaka medical college Hospital, Dhaka. over a period of 02 years from January 2016 to January 2018. Acute Coronary Syndrome was diagnosed by clinical history, ECG evaluation and Serum Cardiac enzyme Troponin I and CK-MB estimation.
\end{abstract}

Result: Most of the patients (73\%) had a sedentary lifestyle and 50\% had dyslipidemia. 40\% patients were taking a diet rich in fats, 37\% patients were diabetic, 33\% were hypertensive, 37\% patients consume oral tobacco, 30\% were smokers, 13\% were obese and 17\% were alcoholics. The family history of premature coronary artery disease was present in only $13 \%$ of cases. In our study, none of the patients had consumed oral contraceptive pills.

Conclusion: The most important modifiable risk factor is the sedentary lifestyle. The commonest non-modifiable risk factor is Male sex. Smoking, oral tobacco consumption, hypertension, diabetes, dyslipidemia, and diet modification are other important modifiable risk factors in young adults.

Keywords: Acute Coronary Syndrome, Cardiac Enzyme, Dyslipidemia. Risk Factors.

J Dhaka Med Coll. 2019; 28(1) : 3-6

\section{Introduction:}

Coronary artery Disease is now the most important and leading cause of death throughout the world ${ }^{1}$. Global burden of disease study estimates that by the year 2020, the burden of atheroembolic cardiovascular disease in this subcontinent would surpass that in any other region in the world ${ }^{2}$.

ACS at an early age constitutes an important problem for the patient as well as the consulting physician as it has devastating effects on the quality of life and survival of young adults.

For better management of this specific problem, it is important to evaluate the risk factors in detail and try to modify them ${ }^{3}$. Studies show a 2 to $10 \%$ incidence of AMI in patients age 45 years or younger ${ }^{4}[3-7,10]$

As very little data is currently available regarding the prevalence of different risk factors for ACS in our younger population, this study on risk factors in young patients of Acute Coronary Syndrome was planned which would help in better management and prevention of ACS in this age group.

\section{Method:}

This is a prospective study which was carried out in 60 young patients (18-40 years of age)

1. Dr. Prodip Kumar Biswas, Associate Professor of Medicine, Dhaka Medical College, Dhaka

2. Dr. Md. Hafiz Sardar, Professor, Department of Medicine, Dhaka Medical College.

3. Dr. Gopal Chandra Saha, Assistant Professor, Department of Radiology and Imaging, Institute of Child and Mother Health, Dhaka.

4. Dr. Mohammad Zaid Hossain, Associate Professor, Department of Medicine, Dhaka Medical College.

5. Dr. Mohammed Shahadat Hossain, Assistant Professor of Medicine, OSD, DGHS. Attached to Faridpur Medical College, Faridpur

6. Dr. Tushit Paul, Honorary Medical Officer, Dept. of Medicine, Dhaka Medical College, Hospital, Dhaka

7. Prof. Khan Abul Kalam Azad, Professor \& Head, Department of Medicine, Dhaka Medical College, Hospital, Dhaka Correspondence: Dr. Prodip Kumar Biswas, Associate Professor of Medicine, Dhaka Medical College, Dhaka Mobile: 01816828895. Email: prodipaurko@gmail.com 
with a diagnosis of Acute Coronary Syndrome admitted to Dhaka medical college Hospital, Dhaka. Bangladesh over a period of 02 years from January 2016 to January 2018. A detailed history was taken in each patient and information of every patient was recorded in a separate proforma. Informed consent was obtained from all patients. The serum cardiac enzyme level was measured, and ECG was done at the time of admission and repeated as necessary.

\section{Results}

The results thus obtained showed that out of 60 patients studied only 18 patients were below 30 years of age, rest $70 \%$ of patients fall between 31- 40 years of age. Mean age was 35 years. There was a male preponderance with $76.67 \%$ males and only $23.33 \%$ females.

Table-I

Risk Factors in the present study

\begin{tabular}{llcc}
\hline S.No. & Risk factors & $\begin{array}{c}\text { No.of } \\
\text { patient }\end{array}$ \\
\hline 1 & Male sex & 46 & $77 \%$ \\
2 & Positive Family History & 08 & $13 \%$ \\
3 & Obesity & 08 & $13 \%$ \\
4 & Diabetes & 22 & $37 \%$ \\
5 & Sedentary lifestyle & 44 & $73 \%$ \\
6 & Diet rich in saturated & 24 & $40 \%$ \\
& fats & & \\
7 & Dyslipidemia & 30 & $50 \%$ \\
8 & Alcohol consumption & 10 & $17 \%$ \\
9 & Smoking & 18 & $30 \%$ \\
10 & Hypertension & 20 & $33 \%$ \\
11 & Oral Tobacco addiction & 22 & $37 \%$ \\
12 & Oral contraceptive pill & 0 & $0 \%$ \\
\hline
\end{tabular}

Most of the patients (73\%) had a sedentary lifestyle and $50 \%$ had dyslipidemia. $40 \%$ patients were taking a diet rich in fats, 37\% patients were diabetic, 33\% were hypertensive, $37 \%$ patients consume oral tobacco, $30 \%$ were smokers, $13 \%$ were obese and $17 \%$ were alcoholics. The family history of premature coronary artery disease was present in only $13 \%$ of cases. In our study, none of the patients had consumed oral contraceptive pills.

Above findings indicate that most common risk factors are a sedentary lifestyle, dyslipidemia, and male sex.

Table-II

Relative frequency of no. of risk factors

\begin{tabular}{lcc}
\hline No. of risk factors & $\begin{array}{c}\text { No. of } \\
\text { patient }\end{array}$ & $\begin{array}{c}\text { Relative } \\
\text { frequency }\end{array}$ \\
\hline $0-2$ & 10 & $16.6 \%$ \\
$3-5$ & 32 & $53.33 \%$ \\
$6-9$ & 18 & $30 \%$ \\
$10-12$ & 0 & $0 \%$ \\
\hline
\end{tabular}

This relative frequency of risk factors is further illustrated in Table 2, which shows that most of the patients, i.e., $53.33 \%$ had $3-5$ risk factors contributing to ACS.

In our study, $27 \%$ of patients had unstable angina, $63 \%$ of patients had ST-elevation myocardial infarction, $10 \%$ of patients had nonST elevation myocardial infarction. The most common type of ACS in young patients is ST elevation MI. Two patients were expired during the hospital stay.

\section{Discussion}

In our study, the observations made in 60 patients of Acute Coronary Syndrome are discussed and compared with other studies.

The youngest age of a patient with ACS in this study was 21 years and the oldest was 40 years. The majority of ischemia/ infarct occurred in the age group between $31-40$ years and only 18 patients were below $30 \mathrm{yrs}$ of age. This correlates with the observation made by Tamrakar $\mathrm{R}$ et $\mathrm{al}^{3}$.

In our study $77 \%$ of patients were male. This finding is consistent with the study of Akhtar et al, who showed $85 \%$ of their patients as a male in their study ${ }^{5}$.

A family history of CAD has an increased risk of premature coronary events. The present study showed that $13 \%$ of patients had a family history of premature CAD, whereas Akhtar et $\mathrm{al}^{5}$ showed $57 \%$ and Tamrakar $\mathrm{R}$ et $\mathrm{al}^{3}$ showed 
$33 \%$ patients having a family history of premature CAD. This difference between the results is probably because of the difference in sample size selected. It is 109 patients in Akhtar et al study ${ }^{5}, 100$ patients in Tamrakar $\mathrm{R}$ et $\mathrm{al}^{3}$ study and 60 patients in our study

We found $73 \%$ of our patients sedentary, which is a significant number, keeping in view the younger population we selected for the study. This is in contrast with Abdul wajid et $\mathrm{al}^{1}$ study in which only $26 \%$ of patients were sedentary but correlates with Deshpande et al study ${ }^{6}$ in which sedentary lifestyle was significantly associated with the risk of ACS. This indicates that lifestyle modification is one of the most common modifiable risk factors for ACS.

We found $37 \%$ of the patients to be diabetic in our study. Curren PJ et al reported $35.7 \%$ of their patients to be diabetic in their study ${ }^{7}$. The results of our study relate very closely to the Akhtar et al study because they also selected patients below 40 years of age,

In present study $33 \%$ of patients were hypertensive. However, the study conducted by Akhtar et al in 1993 on young patients of IHD found $47.6 \%$, hypertensive patients. ${ }^{1}$ This difference is probably because Akhtar et al study included all the patients with IHD whereas our study included only those patients who had ACS, but not stable angina. Another reason is that obesity has also contributed to hypertension in Akhtar et al study, as almost half of their patients were obese whereas most of our patients belonged to poor socioeconomic status and only $13 \%$ were obese.

Dyslipidemia is one of the major modifiable IHD risk factors. Akhtar et al reported dyslipidemia in their $63.2 \%$ of patients. In our study $50 \%$ of patients had dyslipidemia. Sedentary lifestyle seems to be contributing to the increased prevalence of dyslipidemia in our population.

In the present study $30 \%$ of patients were cigarette smokers. Many other studies have also found high rates of smoking among young patients who have Acute myocardial infarction, with percentages ranging from $70 \%$ to $>90 \%$.

All these pieces of evidence point out smoking as an important modifiable risk factor and preventable cause of CAD in young adults. ${ }^{1,3}$ So we support the conclusion that tobacco control programs could have an important contribution in preventing and decreasing the incidence of ACS in our young adults.

In our study, $37 \%$ of patients had oral tobacco addiction. Not many studies were done previously on the relation between oral tobacco addiction and the development of ACS. But interestingly we found that there is a positive correlation between oral tobacco addiction and developing ACS at young age (37\%). Compared to healthy, age-matched control subjects, young patients with myocardial infarction have a higher percentage of smoking and other coronary artery disease risk factors ${ }^{8}$

Use of OC pills was considered as one among the risk factors in most of the studies, but in the present study, none of the participants has used OC pills. This correlates with the observations made by Waters DD et al. ${ }^{9}$ Thus OCPs intake is not an important risk factor in our society.

Clustering of different risk factors of ACS predisposes the patient to premature CAD. In Chen L et al. ${ }^{10}$ study, $55 \%$ of patients had 3 or more risk factors, whereas $70 \%$ of patients in our study had 3 or more risk factors. The observed difference exists because we studied twelve risk factors against six risk factors studied. Therefore, we conclude that as the number of risk factors increases in an individual, the risk of IHD and Acute Coronary Syndrome also increases, and the patient develops ACS at an early age.

\section{Limitation}

This study was conducted in an urban set up in a tertiary care hospital, but most of the patients are of the low socio-economic group; this is the main confounding factor in our study.

\section{Conclusion}

There are important differences that should be appreciated in young patients (at or below 40 years of age) diagnosed with ACS. The most important modifiable risk factor is the sedentary lifestyle. The commonest non-modifiable risk factor is Male sex. Smoking, oral tobacco consumption, hypertension, diabetes, 
dyslipidemia, and diet modification are other important modifiable risk factors in young adults. Other risk factors such as obesity and family history of premature CAD are also prevalent in young ischemic patients but alcohol consumption and OCPs are not commonly seen in young ACS patients and thus not a major health problem for us. The presence of more than one risk factor particularly three or more risk factors in an individual predisposes to ACS at a relatively younger age. Improving lifestyle is a goal of the first magnitude in these patients. If timely managed, young patients with ACS have favorable in-hospital prognosis

\section{References}

1. Abdul Wajid Khan Faisal, Mohammad Ayub, Tariq Waseem, Rao Shahzad Abdul Tawwab Khan, Syed Sibitul Hasnain. Risk factors in young patients of Acute myocardial infarction. J Ayub Med Coll Abbottabad 2011; 23:12-14.

2. Bahl VK, Prabhakaran D, Karthikeyan G; Coronary Artery disease in Indians. IHJ Nov-Dec 2001.

3. Tamrakar R, Bhatt YD, Kansakar S, et al. Acute Myocardial infarction in young adults: study of risk factors, angiographic features and Clinical outcome. Nepalese Heart Journal 2013; 10:12-16.
4. Morillas P, Bertomeu V, Pabón P, Ancillo P, Bermejo $\mathrm{J}$, Fernández C, Arós F PRIAMHO II Investigators. Characteristics and outcome of acute myocardial infarction in young patients. The PRIAMHO II study. Cardiology. 2007; 107:217-25

5. Akhtar J, Islam N, Khan J. Risk factors and outcome of ischemic heart disease in young Pakistani adults. Specialist 1993; 9:123-6.

6. Deshpande J. D. and Dixit J. V. Risk factors for acute myocardial infarction: A Hospital based Case control study health and population - Perspectives and issues 2008; 31:164-169.

7. Curren PJ, Chung EH, Chauhan MS. Metabolic syndrome: an under recognized risk factor for myocardial infarction in the young. J Am Coll Cardiol. 2004;43:24.

8. Moret P, Gutzwiller F, Junod B. Coronary artery disease in young adults under 35 years old: risk factors. pp. 17-22

9. Waters DD, Halphen C, Theroux P, Paul-Robert D, Mizgala HF. Coronary artery disease in young women: clinical and angiographic features and correlation with risk factors. Am J Cardiol. 1978 Jul;42:41-7.

10. Chen L, Chester M, Kaski JC. Clinical factors and angiographic features associated with premature coronary artery disease. Chest. 1995;108:364. 